\title{
Identification of Functional Genic Components of Major Fusarium Head Blight Resistance Quantitative Trait Loci in Wheat Cultivar Sumai 3
}

\author{
Yongbin Zhuang, Aravind Gala, and Yang Yen \\ Department of Biology and Microbiology, South Dakota State University, Brookings, SD 57007, U.S.A.
}

Submitted 1 October 2012. Accepted 3 December 2012.

\begin{abstract}
Fusarium head blight (FHB) is a devastating disease worldwide, affecting wheat and other small grains. To identify key wheat genes involved in FHB pathogenesis, 406 FHBrelated wheat expressed sequence tags functionally identified in Sumai 3 were investigated for their association with FHB-resistance quantitative trait loci (QTL) Fhb1 and $F h b \_6 B L$ in 2010 and 2011. A total of 47 candidate genes were identified by bulk analysis, near-isogenic screening and expression QTL mapping, and were finally mapped to their carrier chromosomes with Chinese Spring nulli-tetra deficiency lines. One gene, designated WFhb1_c1 (wheat Fhb1 candidate gene 1), was both functionally associated with and physically located within $F h b 1$ and was found to be weakly similar $(E=5 e+0)$ to an Arabidopsis gene encoding pectin methyl esterase inhibitor. Two other genes, designated WFI_6BL1 and WFI_6BL2 (wheat-Fusarium interaction genes $6 B L 1$ and $6 B L 2$ ), were functionally associated with $F h b \_6 B L$ but physically mapped on chromosomes 7D and $5 \mathrm{~A}$, respectively. WFI_6BLI was annotated as a 13lipoxygenase gene and WFI_6BL2 might encode a PR-4like protein. Our data suggested that i) Fhb1 seems to contribute to FHB resistance by reducing susceptibility in the first $60 \mathrm{~h}$, ii) $F h b \_6 B L$ makes its contribution via the jasmonate-mediated pathways, and iii) wheat seems to activate its defense mechanism in the biotrophic phase of FHB pathogenesis.
\end{abstract}

Fusarium head blight (FHB, also called scab or head scab), which is caused primarily by the ascomycetous fungus Fusarium graminearum Schwabe [teleomorph Gibberella zeae (Schwein) Petch], is a destructive and economically important disease of wheat and barley. Reportedly, \$1 billion of economic loss has been caused by this disease in relation to wheat alone (McMullen et al. 1997; Parry et al. 1995). Infection mostly occurs in warm and humid or semihumid areas worldwide. The fungus infects hosts by way of asexual conidia or sexually derived ascospores, dispersed largely by wind or rain (Gilbert and Fernado 2004). Germinated spores develop hyphae on the exterior surface of florets and glumes and gain access through stomata and other sites within the inflorescence that

Corresponding author: Y. Yen; E-mail: yang.yen@sdstate.edu; Telephone: $+1605.688 .4567$

*The $\boldsymbol{e}$-Xtra logo stands for "electronic extra" and indicates that two supplementary tables are published online.

(C) 2013 The American Phytopathological Society are susceptible to penetration (Bushnell et al. 2003). Following penetration, Fusarium hyphae can spread within the cell apoplast, leading to significant cytological alterations and, ultimately, to cell death. In addition to lower yield caused by FHB, losses could also come from the contamination of grains with mycotoxins produced by Fusarium graminearum (Desjardins and Hohn 1997; Snijders1990), which is harmful to humans and livestock (Desjardins and Hohn 1997).

Two types of FHB resistance are widely accepted, resistance to the initial infection (type I) and resistance to the spread of infection within the head (type II) (Schroeder and Christensen 1963). Type I resistance resource is rare and is most likely contributed by spike morphology (Mesterhazy 1995) and, possibly, also by activation of systemic innate immune response (Foroud et al. 2012). Type II resistance is controlled by resistance genes and, thus, is the better utilized and studied of the two. Numerous genetic studies with various resistance sources have shown that type II resistance in each resistant wheat cultivar is most likely controlled by two to three major genes plus some minor ones (Gao et al. 2005; Van Ginkel et al. 1996). Molecular mapping of quantitative trait loci (QTL) for type II resistance has been extensively reported. Buerstmayr and associates (2009) summarized that a total of 52 QTL were reported from different resources and were mapped on to all but wheat chromosomes 7D. Of the 52 QTL, the one located on chromosome arm 3BS (termed Fusarium head blight 1 or Fhbl) is the most effective and the most stable across different genetic backgrounds and various environments (Anderson et al. 2001; Basnet et al. 2012; Buerstmayr et al. 2002; Somers et al. 2003; Zhou et al. 2002). Fhb1 was first identified from the Chinese cultivar Sumai 3 and could explain up to $60 \%$ of the total phenotypic variation (Buerstmayr et al. 2009).

Sumai 3 is one of the most FHB-resistant wheat cultivars and has been extensively used as a major FHB-resistance source in breeding programs worldwide. However, a major problem with using Sumai 3 in improving FHB resistance is that its FHB resistance is almost always tightly associated with other unfavorable agriculture traits, including low grain yield and, especially, susceptibility to other diseases, such as powdery mildew and leaf rust (Wilde et al. 2007). This tight association was believed to be caused by linkage drag (Cuthbert et al. 2007) or by the QTL $\times$ genetic background interaction effect (Pumphrey et al. 2007), but it could also be due to the nature of multiple functions of the genes associated with Fhbl. Therefore, identifying and cloning one or more functional genes associated with Fhbl and studying their molecular functions are necessary. Fine mapping has narrowed Fhbl down to a 261-kb region on 3BS (Bernardo et al. 2012; Liu et al. 2008; Zhou et al. 2010), and seven potential genes in this QTL re- 
gion have been cloned and analyzed (Liu et al. 2008). However, no functional gene in Fhbl or any other QTL has been reported.

Several transcriptome studies have been conducted and revealed tens or even hundreds of differentially expressed genes between a resistant and a susceptible material that could be involved in wheat $-F$. graminearum interaction (Bernardo et al. 2007; Cho et al. 2012; Gottwald et al. 2012; Jia et al. 2009; Kruger et al. 2002; Li and Yen 2008; Trail et al. 2003). Although variant genetic backgrounds of the studied materials have made cross comparison among the results very difficult, the studies by $\mathrm{Li}$ and Yen (2008) and Gottwald and associates (2012) did agree that it is the signaling pathways mediated by jasmonate (JA) and ethylene (ET) that control FHB resistance in wheat.

Our previous studies of FHB resistance in Sumai 3 identified a few hundreds of differentially expressed wheat genes that were associated with FHB resistance (Li and Yen 2008) and were mapped two major FHB-resistance QTL (Basnet et al. 2012). In this communication, we report our work to associate these differentially expressed genes with the mapped QTL by expression (e)QTL analysis. A pair of bulked recombinant inbred line (RIL) pools and a pair of near-isogenic lines (NIL) for Fhbl were used for preliminary screening. Expression of the identified genes was used as a phenotypic trait to associate the gene with a mapped QTL. A gene associated with a QTL by the eQTL analysis can be either cis acting (a functional gene of the QTL) or trans acting (a downstream gene directly controlled by the QTL) (Brem et al. 2002; Yamashita et al. 2005). Therefore, aneuploidy analysis was conducted to confirm the physical location of the identified genes in the wheat genome. This gene-QTL association should facilitate identification and cloning of one or more functional components of the mapped FHB resistance QTL, helping understanding one or more resistance mechanisms.

\section{RESULTS}

Our general strategy to identify candidate genes for the mapped FHB resistance QTL is illustrated in Figure 1. Generally, this strategy consisted of preliminary screening with RIL bulk analysis, eQTL analysis to identify candidate genes, and physical mapping of the candidate genes. NIL analysis was also conducted to confirm gene association with Fhbl. With this strategy, we were able to identify one candidate gene for $F h b 1$ and two genes controlled by the QTL on chromosome arm 6BL.

\section{Bulk analysis of expressed sequence tags (EST) potentially associated with mapped QTL.}

A resistant and a susceptible RIL bulk were formed by pooling 10 RIL per bulk from a $F_{2: 8}$ Sumai 3/Y1193-06 RIL mapping population and were used for preliminary screening of potential candidate genes for the mapped FHB resistance QTL. Y1193-06 is a landrace from Tibet and is the most susceptible wheat line to FHB we have ever tested. Sumai 3 and Y1193-06 were the materials used in our microarray assay that identified FHB-associated wheat genes (Li and Yen 2008). The RIL mapping population was used in our identification of two major FHB-resistance QTL on chromosome arms 3BS and 6BL in Sumai 3 and a major FHB-susceptibility QTL on 2DS probably from Y1193-06 with the aid of simple sequence repeat and DArT markers (Basnet et al. 2012). The RIL were selected on the basis of their extreme performance in either resistance or susceptibility in the RIL population.

A total of 406 wheat genes were screened for their differential expression, first within each bulk and then between the bulks, in 2010 and 2011. The screening within the bulks enabled us to identify genes that responded to the pathogen infection, while the screening between the bulks resulted in identification of the genes that might be associated with FHB-resistance.

Our results showed that 121 and 86 genes showed differential expression between the two bulks in 2010 and 2011, respectively. Of them, 47 were common in both years and were considered to be potential candidate genes for further analysis. These 47 genes were grouped into nine clusters according to their functional annotation (Supplementary Table 1). Of these 47 genes, 14 were specific to the resistant bulk, 13 were specific to the susceptible bulk, and 20 were shared by the two bulks.

Expressions of these 47 EST were then analyzed in the two years, using the two NIL for their association with $F h b 1$. This screening identified three potential candidate genes (BE415640, CA665159, and CA640991) by their significantly differential expression between the two NIL. The remaining 44 genes were considered as potential candidate genes for the other two QTL.

\section{Identifying candidate genes}

to the mapped QTL by eQTL analysis.

Our previous QTL mapping, which led to the identification of two major FHB-resistance QTL from Sumai and one major FHB-susceptible QTL from Y1193-06, were conducted with $160 \mathrm{~F}_{2: 6}$ Sumai 3/Y1193-06 RIL (Basnet et al. 2012). To save time and reduce expenses, $80 \mathrm{~F}_{2: 8}$ RIL derived from the $\mathrm{F}_{2: 6}$ RIL were used in this study instead. The selection was based on their performance over years. To verify that these 80 RIL were indeed able to truly represent the entire RIL population, they were investigated for FHB disease index (DI) and number of Fusarium-infected kernels (FDK) in 2010 and 2011. The data were used to conduct QTL mapping, and all of the three QTL previously identified with the entire RIL population were detected (Fig. 2) with these 80 RIL. This result confirmed that the 80 RIL could represent the entire RIL population; hence, they were used for eQTL analysis.

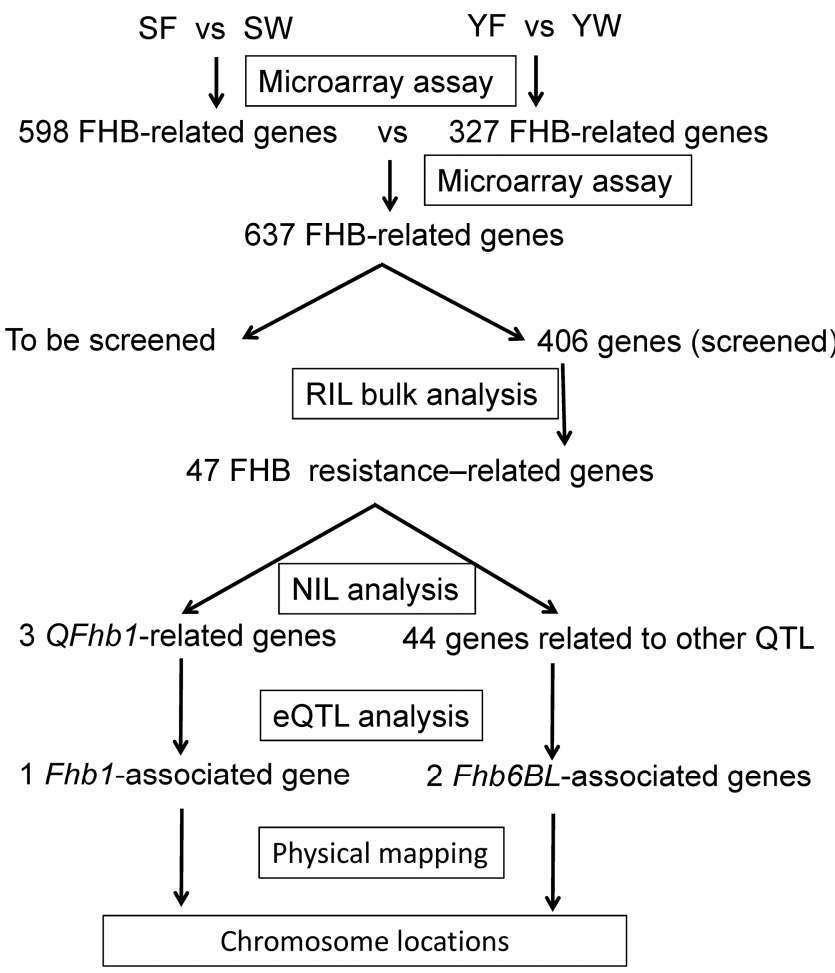

Fig. 1. General strategy for identifying candidate genes for major Fusarium head blight (FHB)-resistance quantitative trait loci (QTL). SF: FHBinoculated Sumai 3; SW: water-inoculated Sumai 3; YF: FHB-inoculated Y1193-06; YW: water-inoculated Y1193-06. RIL = recombinant inbred lines; NIL $=$ near-isogenic lines. 
Expressions of the 47 potential candidate genes at $24 \mathrm{~h}$ after inoculation (hai) were assayed with quantitative polymerase chain reaction (qPCR) in 2010 and 2011 for eQTL analysis. Of the 47 genes, 29 were mapped to a total of 71 chromosome locations when the log of the likelihood ratio (LOD) $>2.5$ was used. The results are listed in Supplementary Table 2. In summary, 23 were in the A genome, 32 in the B genome, and 16 in the D genome. Only chromosomes 4B, 5D, and 6D did not host any. Nine genes were located only on one chromosome and five only on two chromosomes. On the other hand, three genes were located on four chromosomes and two on five chromosomes. Of the 71 eQTL, 62 were detected in both years. Clearly, reproducibility across the two-year analysis was quite good. After combining those that were located within 2 centiMorgans (cM) from each other, a total of 57 eQTL were mapped on 16 wheat chromosomes. Figure 3 showed the distribution of the detected eQTL across the wheat genome. Obviously, chromosome $3 \mathrm{~B}$ had the most eQTL, followed by chromosomes $6 \mathrm{~B}, 1 \mathrm{D}$, and $1 \mathrm{~B}$.

Of the three genes associated with Fhbl by NIL screening, only two (BE415640 and CA640991) were mapped on 3B by the eQTL mapping. Background interference could be a possible explanation. Interestingly, CA640991 was mapped exactly to the same chromosome interval as $\mathrm{Fhbl}$ did on chromosome arm 3BS (Fig. 2A). Therefore, the corresponding gene was likely a component of this QTL and, thus, was designated as WFhbl_cl (wheat Fhblcandidate gene 1). The other two Fhbl-associated genes (BE415640 and CA665159) that were not mapped to $F h b 1$ seemed to be functionally controlled by Fhbl and were, therefore, assigned as WFhbl_ml and
WFhb1_m2 (Wheat Fhb1-mediated genes 1 or 2). Two other genes, which were designated as WFI_6BL1 and WFI_6BL2 (wheat- $F$. graminearum interacting genes $6 B L 1$ or $6 B L 2$ ), were similarly mapped to the same chromosome interval on chromosome arm 6BL as the mapped QTL Fhb_6BL (Fig. 2B and C). The three genes accounted for up to 39,25 , or $32 \%$ of the phenotypic variance in the two-year trial (Table 1).

We also conducted correlation analyses between the expression changes of WFhb1_c1, WFI_6BL1, or WFI_6BL2 and FDK in the two years, respectively (Table 2). Only WFhbl_cl

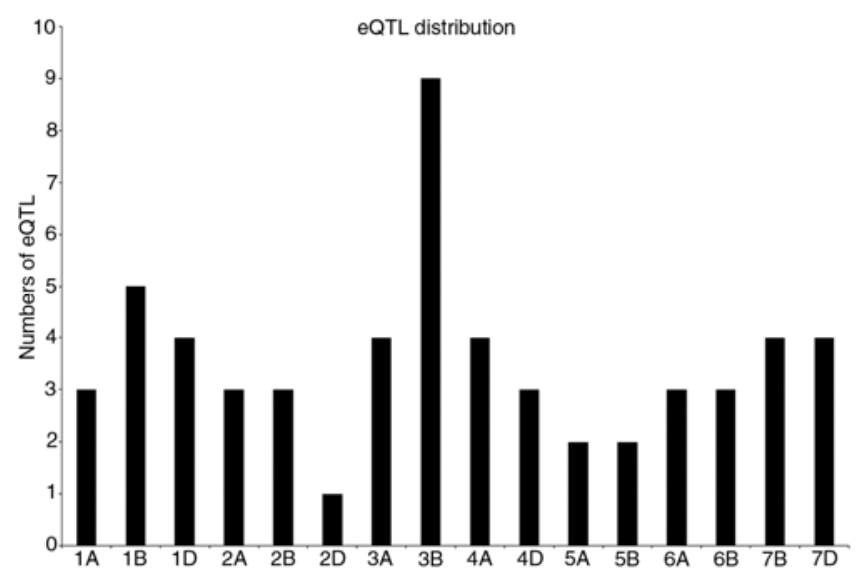

Fig. 3. Distribution of Fusarium head blight (FHB)-related expression resistance quantitative trait loci (QTL) across the wheat genome.
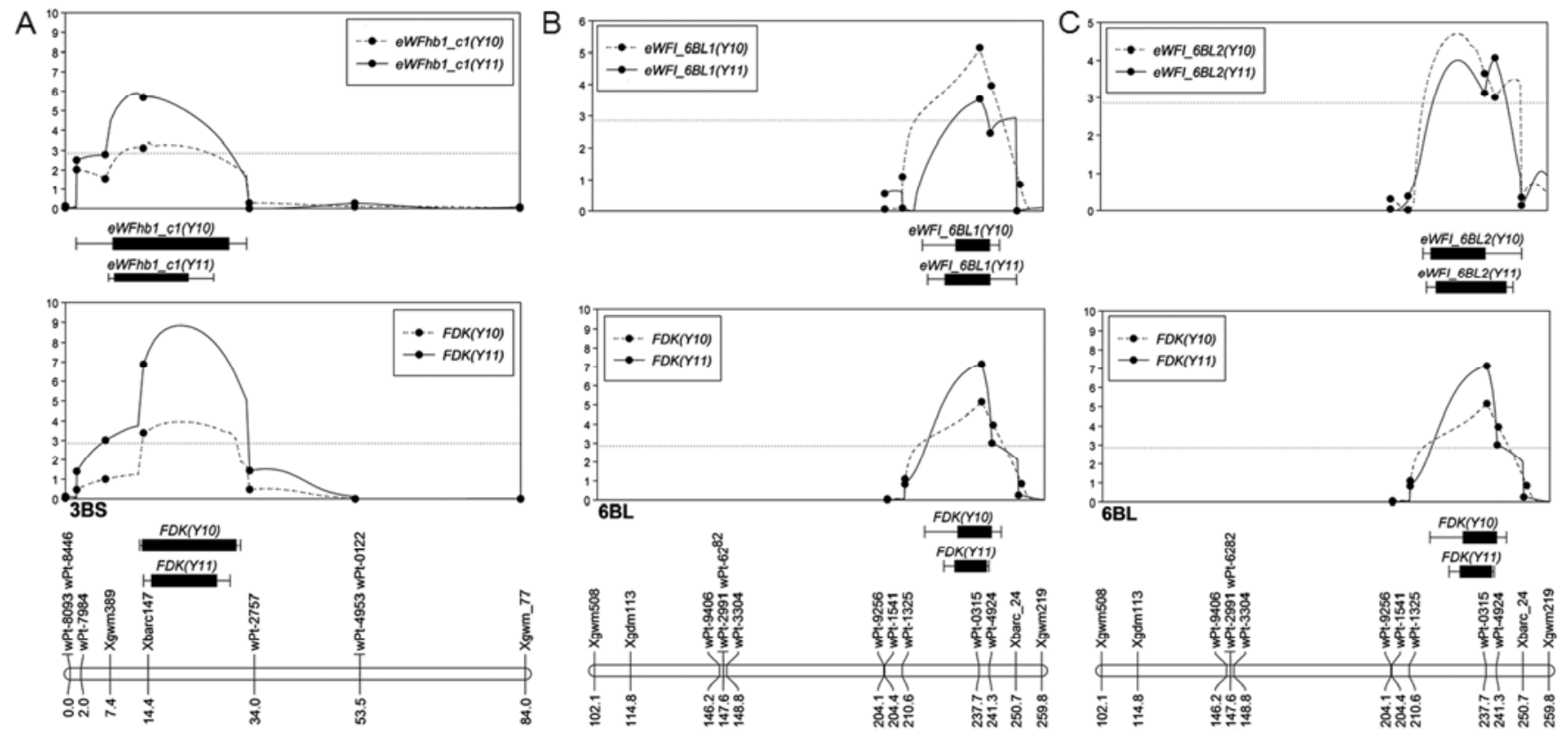

Fig. 2. Chromosome locations of Fusarium head blight (FHB)-resistance quantitative trait loci (QTL) Fhb1 and Fhb_6BL and expression (e)QTL eWFhb1_c1, eWFI_6BL1 and eWFI_6BL2. A, QTL Fhb1 and eQTL eWFhb1_c1; B, QTL Fhb_6BL and eQTL eWFI_6BL1; C, QTL Fhb_6BL and qQTL eWFI_6BL2. FDK: Fusarium diseased kernel; Y10: 2010; Y11: 2011.

Table 1. Characteristics of candidate genes associated with mapped quantitative trait loci (QTL)

\begin{tabular}{lllccccc}
\hline Year & Gene & Closest markers & QTL associated with & Chr arm & eQTL position (cM) & LOD & Variance explained (\%) \\
\hline 2010 & WFhb1_c1 & Xbarc147 & Fhb1 & $3 B S$ & 14.40 & 3.37 & 24.59 \\
& WFI_6BL1 & Wpt0315 & Fhb_6BL & $6 \mathrm{BL}$ & 237.71 & 5.15 & 24.91 \\
& WFI_6BL2 & Wpt0315 & Fhb_6BL & $6 \mathrm{BL}$ & 227.13 & 4.70 & 11.15 \\
2011 & WFhb1_c1 & Xbarc147 & Fhb1 & 3BS & 13.41 & 5.71 & 38.95 \\
& WFI_6BL1 & Wpt0315 & Fhb_6BL & $6 \mathrm{BL}$ & 237.71 & 3.54 & 22.23 \\
& WFI_6BL2 & Wpt0315 & Fhb_6BL & 6BL & 227.61 & 4.00 & 31.93 \\
\hline
\end{tabular}


showed a very significant $(P \leq 0.0089$ in year 2011$)$ or significant $(P \leq 0.0274$ in year 2010$)$ negative correlation with FDK in the two-year trial, suggesting that a lower WFhbl_cl expression level was associated with more severe disease. Correlations of FDK with the other two genes, although insignificant, were both positive.

Sequence analysis revealed that WFI_6BL1 might encode a 13-LOX (lipoxygenase) and WFI_6BL2 seems to be a gene of a PR-4 family protein. The exact function of WFhbl_cl remains unknown. However, it was found to have no homology with any known wheat gene and was only very weakly similar $(E=5 \mathrm{e}+0)$ to an Arabidopsis pectin methyl esterase inhibitor (PMEI). WFhbl_ml was weakly similar $(E=5 \mathrm{e}+0)$ to an Arabidopsis gene encoding a sec-independent translocation protein Hcfl06 (high chlorophyll fluorescence 106), and WFhb1_m2 was found to be a Pox family gene $(E=0)$, encoding a peroxidase family protein.

\section{Identifying the physical locations}

of the candidate genes in wheat genome.

An eQTL can be cis- or trans-acting, and only a cis-acting eQTL can be a functional gene component of a QTL. Therefore, physical mapping of the three candidate genes using Chinese Spring nulli-tetra lines was performed to determine whether they are trans-acting or cis-acting genes. A nulli-tetra line is an aneuploidy line in which a pair of a chromosome has been replaced by a pair of its homoeologous chromosomes. For instance, both copies of chromosome $3 \mathrm{~B}$ were missing from the nulli 3B-tetra 3A line, which has four copies of chromosome $3 \mathrm{~A}$. As a result, all genes on the replaced chromosomes will be missing from the line. As shown in Figure 4, PCR failed to amplify the WFhbl_cl amplicon from the nulli 3Btetra 3A line. Therefore, $\bar{W} F h b 1 \_c 1$ has been physically mapped to chromosome 3B and, thus, was a cis-acting gene of Fhb1. It seems that chromosomes $2 \mathrm{D}$ and $3 \mathrm{~A}$ also have a smaller-sized homoeologous copy of this gene amplicon (Fig. 4 ). Whether these two homoeologous copies are functional or not remains unknown, since our eQTL mapping has only located WFhb1_cl onto 3B. Similarly, WFI_6BL1 was physically mapped to chromosome 7D and WFI_6BL2 to chromosome 5A (Fig. 4). Therefore, they are both tans-acting genes partially controlled by FHB-resistance QTL Fhb_6BL. WFI_ $6 B L 1$ primer sets amplified two bands, both from chromosome $7 \mathrm{D}$, and WFI_6BL2 seemed to have only one locus in the wheat genome (Fig. 4).

\section{Investigation of gene expression dynamics during wheat-Fusarium interaction.}

Expression patterns of the three candidate genes that were mapped to the two FHB-resistance major QTL in Sumai 3 were also assayed at 4, 8, 12, 24, and 48 hai by qPCR to investigate their behaviors in both Sumai 3 and Y1193-06 during the early stage of the wheat $-F$. graminearum interaction. The two NIL were also assayed for the expression of WFhbl_c1 from 12 to 96 hai. The results are shown in Figure 5. Generally, expressions of the two candidate genes associated with $F h b \_6 B L$ both had an increased phase followed by a suppression phase, with WFI_6BL1 peaking $16 \mathrm{~h}$ earlier in both resistant and susceptible genotypes. WFI_6BL1 had a similar expression pattern in both genotypes across the entire time frame tested, but its expression was always about two- to fourfold higher in Sumai 3 than in Y1193-06 (Fig. 5C). WFI_6BL2 differentiated its expression between the two genotypes starting about 8 hai and ending at about 48 hai, with an 11-fold peak difference at about 24 hai (Fig. 5D). Both WFhbl_cl and WFI_6BL2 showed significant differential expression between Sumai 3 and Y1193-06, but their patterns were just opposite to each other in the two (Fig. 5A, B, and D). While WFhbl_cl had a relatively slight increase in its expression during the course in both resistant lines, the expression in both the sus-

Table 2. Pearson's product-moment correlation analysis of gene expression change and number of Fusarium diseased kernels

\begin{tabular}{lccllc}
\hline Year & Candidate gene & $\boldsymbol{t}$ & df & $\boldsymbol{P}_{\text {value }} \mathbf{a}^{\mathbf{2}}$ & \multicolumn{1}{c}{ Correlation } \\
\hline 2010 & WFhb1_c1 & -2.2805 & 80 & $0.0274^{*}$ & -0.3219 \\
& WFI_6BL1 & 0.4881 & 75 & 0.6285 & 0.0822 \\
& WFI6BL2 & 0.3319 & 79 & 0.7419 & 0.0552 \\
2011 & WFhb1_c1 & -2.6577 & 80 & $0.0089 * *$ & -0.2278 \\
& WFI_6BL1 & 0.5595 & 75 & 0.5794 & 0.0942 \\
& WFI_6BL2 & 0.9188 & 79 & 0.3643 & 0.1514 \\
\hline
\end{tabular}

a $*=$ significant at $P \leq 0.05$ level; $* *=$ significant at $P \leq 0.01$ level.
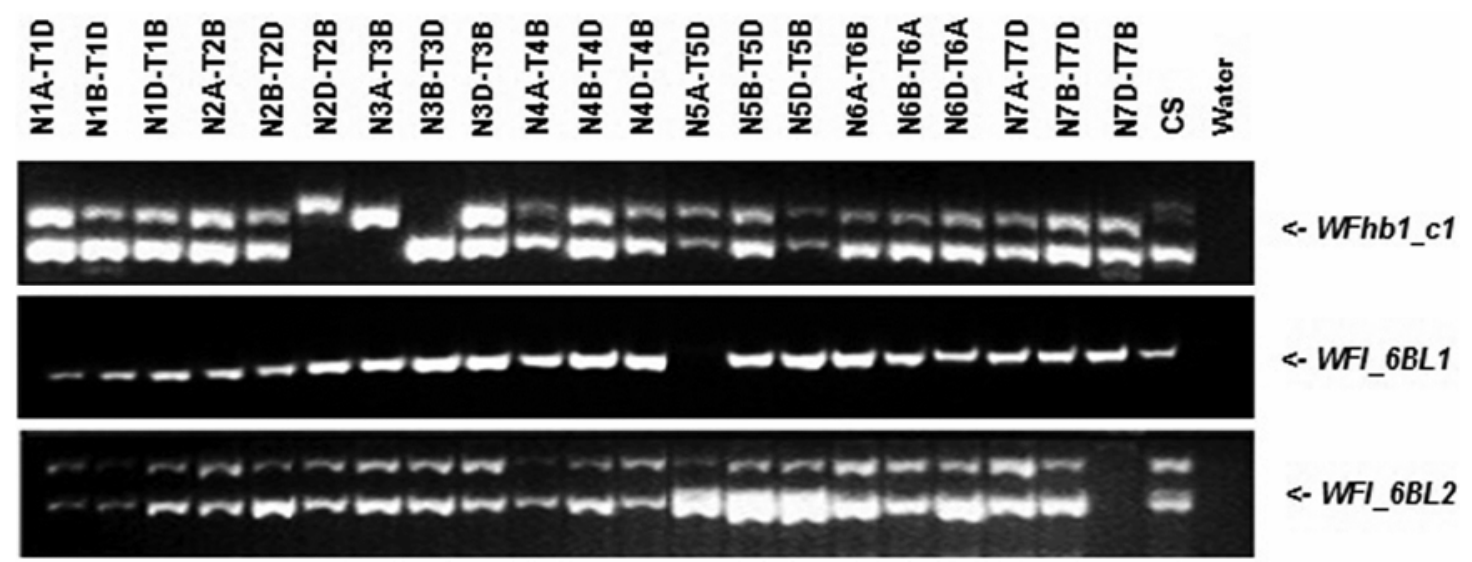

Fig. 4. Results of physical mapping of the candidate genes WFhb1_c1,WFI_6BL1 and WFI_6BL2. The nulli-tetra series (Nx-Ty) of wheat cultivar Chinese Spring (CS) were used. In each line, a pair of chromosomes (Nx) was replaced by an extra pair of its homoeologous chromosomes (Ty). For instance, an extra pair of chromosome 3D replaced a pair of chromosome 3B in the line N3B-T3D. CS was used as the positive control and the negative control has everything the other polymerase chain reactions have but DNA templates. 
ceptible lines had a sharp drop of around 20-fold, seemingly starting from about 8 hai and peaking at about 24 hai, when a recovery was seen (Fig. 5A and $\mathrm{B}$ ). The differential expression of WFhbl_cl between the resistant and the susceptible lines, at least in the NIL, apparently ended at about 60 hai (Fig. 5B).

\section{DISCUSSION}

Chromosome 3B plays an important role in regulating the wheat- $F$. graminearum interaction.

Resistance to FHB in wheat is quantitative and more than 50 resistance QTL have been mapped on to all but wheat chromosome 7D (Buerstmayr et al. 2009). However, only Fhbl on chromosome arm 3BS has been constantly detected in every resistance source and each environment so far investigated. Analyses also found that $F h b 1$ is accountable for 20 to $60 \%$ of the variation for FHB resistance (Anderson et al. 2001; Basnet et al. 2012; Buerstmayr et al. 2002; Somers et al. 2003; Zhou et al. 2002). Of the 47 FHB resistance-related genes investigated, 11 were associated with chromosome $3 \mathrm{~B}$. Nine of the 57 eQTL detected in this study were mapped on 3B (Fig. 3). These FHB resistance-related genes are either physically located on 3B as a cis-acting eQTL or located on other chromosomes but have their expressions controlled by genes on 3B (as a trans-acting eQTL). All these data suggested that chromosome $3 \mathrm{~B}$ plays an important role in regulating the wheat $-F$. graminearum interaction during FHB pathogenesis.
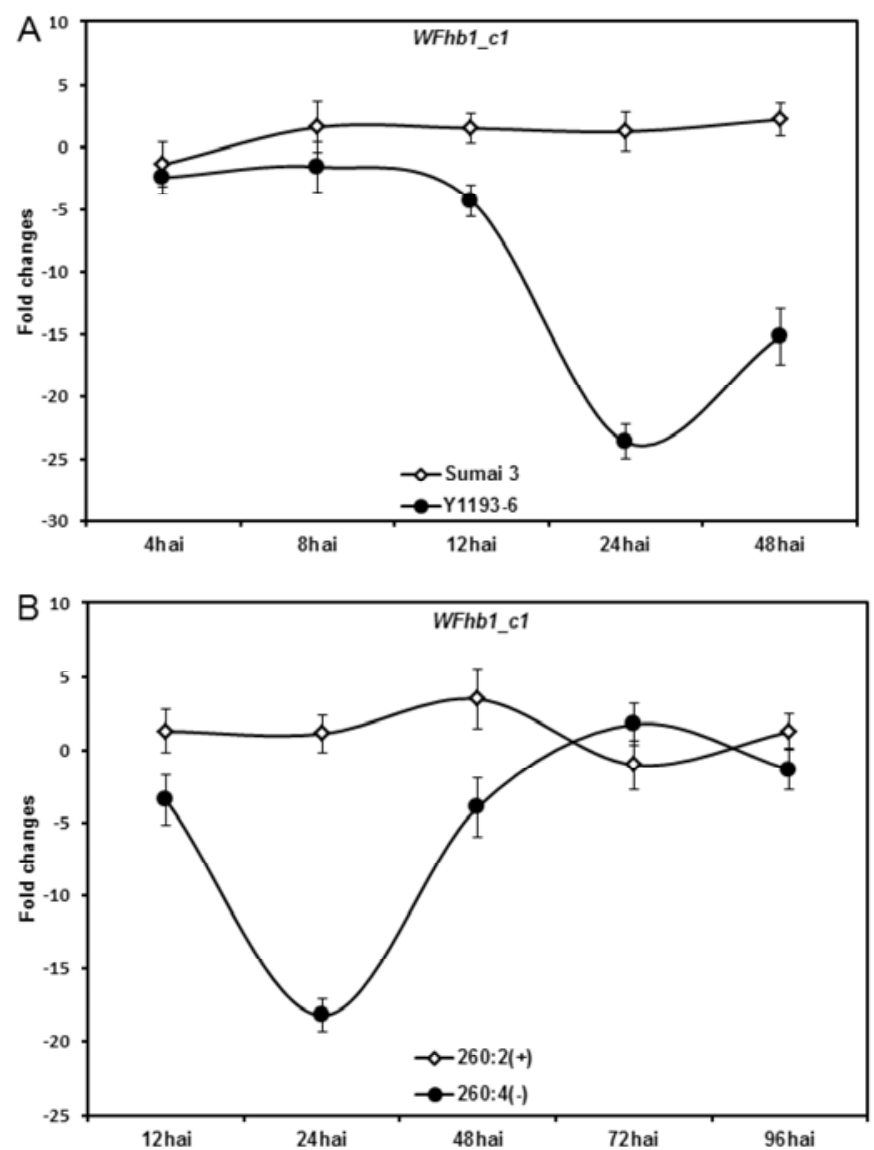

Fhb1 could contribute to delay of FHB spreading by reducing FHB susceptibility

rather than increasing $\mathrm{FHB}$ resistance.

Because of the importance of $F h b l$ in wheat's resistance to FHB, investigations have been made to understand its functions, which resulted in contradictory conclusions. Lemmens and associates (2005) believed that Fhbl's function is detoxifying deoxynivalenol (DON). Jia and associates (2009) did not find any evidence to support the hypothesis that Fhbl contributed resistance to initial DON accumulation. Efforts to clone the functional genes located within $F h b l$ have also been made (Liu et al. 2008; Pumphrey et al. 2007), but none has been reported. Hill-Ambroz and associates (2006) physically mapped an FHB-induced gene for glyceraldehyde 3-phosphate dehydrogenase to the Fhbl region but did not functionally connect the two. Our combined efforts with transcriptomic analysis ( $\mathrm{Li}$ and Yen 2008), genetic studies (Basnet et al. 2012), eQTL assay (Fig. 2A), and physical mapping (Fig. 4) have successfully identified a functional gene, WFhb1_cl, within this QTL, which could explain about 24 to $39 \%$ of the phenotypic variation in our two-year trial (Table 1). A negative correlation of its expression changes with FHB severity within the RIL population was also significant in both years (Table 2). Although we still do not know the exact function of WFhb1_cl, our bioinformatic analysis indicated that it could be a PMEI gene involved in cell-wall modification. Wheat PMEI genes are known to have low sequence conservation (Rocchi et al. 2011). Volpi and associates (2011) reported that
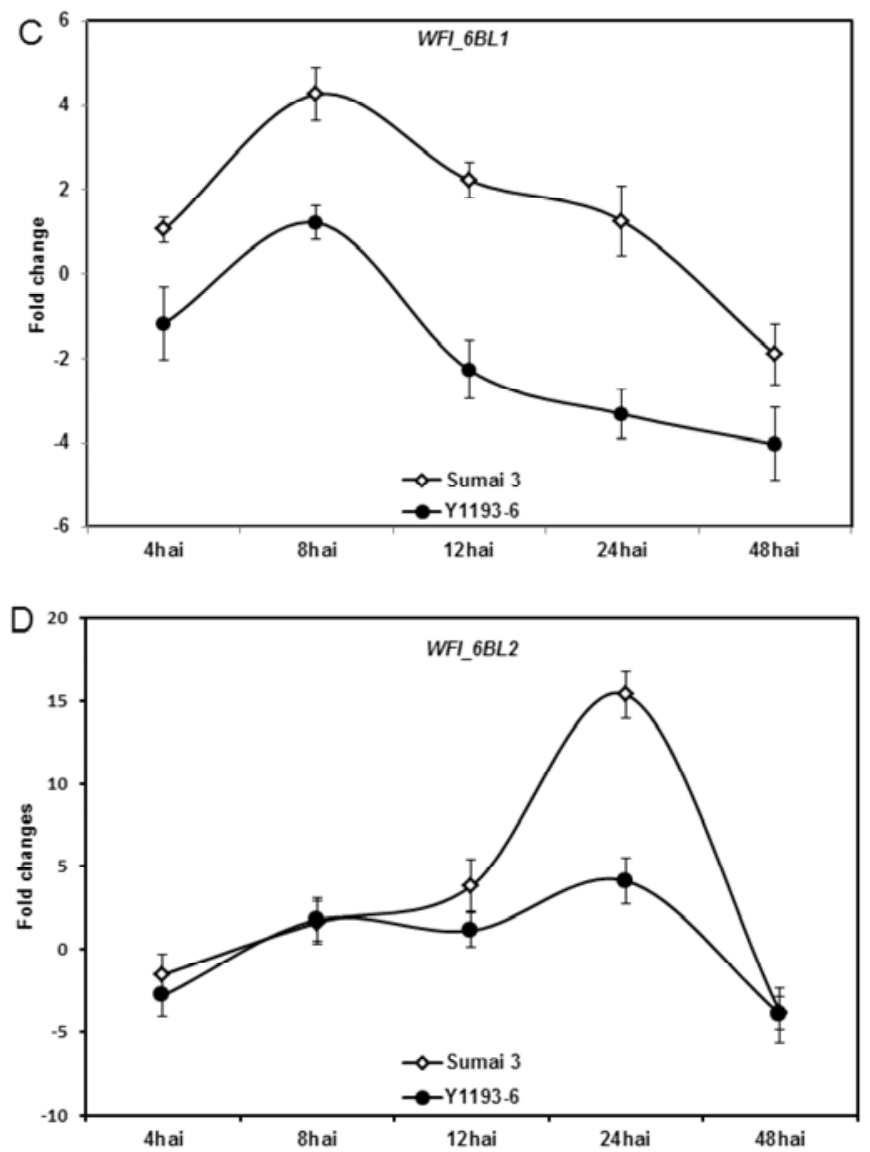

Fig. 5. Expression dynamics of the three candidate genes for major Fusarium head blight (FHB)-resistance quantitative trait loci (QTL) showing as fold changes between the FHB-inoculated and the mock-inoculated samples at hour after inoculation (hai). A, WFhb1_cl in Sumai 3 and Y1193-06; B, WFhb1_c1 in near-isogenic lines Fhb1NILs 260-2 (fhb1+) and 260-4 (fhb1-); C, WFI_6BL1 in Sumai 3 and Y1193-06; D, WFI 6BL2 in Sumai 3 and Y1193-06. Expression was assayed by real-time quantitative polymerase chain reaction with $\beta$-actin as the reference gene. The $2^{-\Delta \overline{\Delta C t}}$ method was used to calculate fold changes between the FHB-inoculated and the water-inoculated samples. If the value of $2^{-\Delta \Delta \mathrm{Ct}}<1$, the negative inverse is taken to calculate the fold change reduction. The error bar shows standard error. 
overexpression of a PMEI from Actinidia chinensis reduced FHB symptoms in transgenic durum wheat.

Pectin-rich middle lamella and cell wall are the physical barriers that a fungal pathogen first encounters during its infection of plant cells. Homogalacturonan (HGA) is a main component of pectin with some methyl side groups. Pectin methyl esterases (PME) function in HGA demethylesterification, enabling aggregation of HGA into a calcium-linked gel structure. However, this action by PME makes HGA susceptible to degradation by hydrolases such as endopolygalacturonases produced by many fungal pathogens (Brummell et al. 2001), which will facilitate the infection process while eliciting defense responses (Bruce and West 1982). F. graminearum genome contains quite a few genes encoding pectin-degrading enzymes (Cuomo et al. 2007). PMEI is a double-edged sword. It can neutralize PME, inhibiting pathogen infection yet reducing production of elicitors to defense response in the host. Studies in potato (Solanum tuberosum) and Arabidopsis have indicated that resistant genotypes to necrotrophic pathogens usually have reduced PME activity (Lionetti et al. 2004; Marty et al. 1997). Research has also found that the three major signaling molecules, salicylic acid, JA, and ET, all can induce PMEI (An et al. 2008). Our data suggested that WFhb1_cl had only a relatively slight increase in expression in FHB-resistant wheat in response to $F$. graminearum infection, but its expression had a sharp, deep suppression between 8 and 24 hai in susceptible genotypes, a susceptible reaction presumably induced by $F$. graminearum infection (Fig. 5A and B). If WFhb1_cl indeed encoded a PMEI, wheat could resist FHB, at least in part, not by increasing resistance reaction (increasing PMEI expression), but by having a much less susceptible reaction to the infection. Based on their transcriptome analysis, Bernardo and associates (2007) hypothesized that FHB resistance in wheat might result from a loss of function of susceptibility genes. Liu and associates (2008) also discovered that a DNA marker close to Fhbl is null, suggesting a deletion in this major FHB-resistance QTL. Both parents of Sumai 3 are susceptible to FHB (He et al. 2001). Therefore, it is possible that WFhbl_cl has lost a regulating site where a suppressor produced or induced by $F$. graminearum or wheat binds.

\section{QTL Fhb_6BL may contribute to disease resistance through the JA-mediated signaling pathway.}

Although our investigation did not discover any gene that is physically located in the $F h b \_6 B L$ region, two genes, WFI_6BL1 and WFI_6BL2, were found to be under the control of this major FHB resistance QTL (Fig. 2B). WFI_6BL1 was found to have about a threefold greater increase in expression in Sumai 3 than in Y1193-06 across the entire time frame we investigated. WFI_6BL1 apparently encodes a 13-LOX, which is a known key enzyme in JA biosynthesis (Creelman et al. 1997; Feussner and Wasternack 2002; Schaller et al. 2004). The average threefold greater expression in Sumai 3 should have led to a much greater increase in JA biosynthesis and, thus, a much stronger JA-mediated resistance response. In fact, this can be evidenced by the behavior of WFI_6BL2 in the two wheat lines. WFI_6BL2 probably encodes a PR-4 protein, which could be induced by JA (Bertini et al. 2003). Our data suggested that WFI_6BL2 peaked its expression about $16 \mathrm{~h}$ later than WFI_6BL1, and its expression level was also much higher in Sumai 3 than in Y1193-06 at the peak (Fig. 5D). Therefore, our data seemed to confirm our previous conclusion that FHB resistance in wheat is mediated by the JA-signaling pathway. It has further suggested that the major FHB-resistance QTL on chromosome arm 6BL may be directly involved in regulating this JA-mediated resistance and WFI_6BL1 works upstream of WFI_6BL2 in the pathway.
It is not surprising that $W F I \_B L 1$ was activated first, then dropped steadily after the expression of WFI_6BL2 had a surge in the FHB-resistant wheat. The first interaction between a fungal pathogen cell and its host cell is the contact between the cell walls of the two. Chitin oligosaccharides in fungal cell wall, after making the contact, can trigger the expression of LOX in the host cells (Doares et al. 1995), leading to an increased JA biosynthesis there. PR-4 proteins can be induced by the JA-signaling pathway. However, the function of PR-4 in plant defense is to neutralize pathogen attack by binding on chitin (Hejgaard et al. 1992). Wheat PR-4 family proteins Wheatin 1 and Wheatin 2 were also known to possess ribonuclease activity and were able to inhibit hypha growth (Bertini et al. 2009), leading to a total reduction in contact between the pathogen and the host. Therefore, the increased expression of WFI_6BL1 possibly triggered the upward surge in expression of the WFI_6BL2 in the FHB-resistant wheat. The product of $W F I 6 B L 2$ then acts to reduce chitin's action on the host cell wall, leading to a reduction in $L O X$ gene expression.

\section{Biotrophic phase of FHB pathogenesis is crucial for wheat to establish resistance to Fusarium infection.}

The three candidate genes we investigated for expression dynamics all showed significant differentiation in their expression in the first one or two days of FHB pathogenesis between the resistant and susceptible genotypes; and the differentiation in all started prior to 12 hai, and all but WFI_6BL1 peaked at about 24 hai (Fig. 5). Clearly, the first $24 \mathrm{~h}$ in FHB pathogenesis is critical to establish a resistant interaction between the host and the pathogen in wheat. Previous observations (Bushnell et al. 2003; Kang and Buchenauer 2000; Pritsch et al. 2000; Seong et al. 2008) showed that FHB pathogenesis has two phases, a biotrophic ( $<72$ hai) phase followed by a necrotrophic ( $>72$ hai) phase. Fusarium macroconidia usually germinate within 5 to $6 \mathrm{~h}$ after landing on wheat spikes. Emerging germ tubes elongate rapidly over glume and floret surfaces and then enter the host cells either through stomata or by direct cell-wall penetration after partially dissolving the surface materials of epidermal cells in adaxial lemma. Subcuticular growth of the pathogen leads to their spreading within the host tissues. Transcriptomic analysis by Kruger and associates (2002) also suggested that $F$. graminearum act as biotrophic fungi at the first 48 hai. Fhbl and $F h b \_6 B L$ were the only major FHB-resistant QTL we detected in Sumai 3 (Basnet et al. 2012). The three candidate genes associated with the two QTL were able to explain a total of up to approximately $90 \%$ of the phenotype variation in our two-year trial (Table 1). In fact, Paranidharan and associates (2008) indicated that the main difference in resistance-related metabolites between resistant and susceptible wheat cultivars occurred $<48$ hai. Therefore, it is highly possible that the biotrophic phase of FHB pathogenesis is what Sumai 3 resists against. In other words, Sumai 3 resists FHB spreading within the infected spikes by delaying the necrotrophic pathogenic development.

In summary, our eQTL study associated three genes with the two major FHB-resistance QTL identified in Sumai 3. $W F h b 1 \_c 1$, which was responsible for up to approximately $40 \%$ of the phenotypic variance in our two-year trial, was both functionally and physically associated with $F h b 1$. Therefore, WFhbl_cl is likely an important component of Fhbl and, thus, a key player in type II resistance. The other two candidate genes seemed to be functionally mediated by $F h b \_6 B L$ but physically located on other chromosomes in the genome. They might contribute to FHB resistance via the JA-mediated resistance pathway. Analysis of expression dynamics of these three candidate genes indicated that the biotrophic phase seems to be critical for wheat to establish its defense mecha- 
nism against $F$. graminearum. Our data also suggested that $\mathrm{Su}-$ mai 3 might resist against FHB by having lost its susceptibility. Cloning and functionally characterizing these three genes should test our hypothesis and facilitate our understanding of FHB pathogenesis and our control of FHB epidemics.

\section{MATERIALS AND METHODS}

\section{Plant materials.}

Wheat (Triticum aestivum L.) cultivar Sumai 3 (FHB resistant), landrace Y1193-06 (FHB-susceptible), and a Sumai 3/Y1194-06 $\mathrm{F}_{2: 8}$ RIL population were used in this study. These RIL were derived from the $\mathrm{F}_{2: 6}$ RIL that we previously used for identifying and mapping major FHB-resistant QTL (Basnet et al. 2012). Sumai 3 and Y1193-6 were also the lines that we studied in our microarray essay for the discovery of $365 \mathrm{FHB}$ resistance-related genes ( $\mathrm{Li}$ and Yen 2008). Two subpopulations were formed by selecting the 10 most-resistant or the 10 most-susceptible RIL lines for bulk analysis. A NIL pair (Fhb1NIL 260-2 and 260-4), which carries either the resistant $(f h b 1+)$ or susceptible $(f h b 1-)$ allele at the Fhbl locus (Pumphrey et al. 2007), was also used in this study. They were provided by J. Anderson of University of Minnesota-Twin Cities. Plants were grown in a greenhouse under a 16-h light and 8-h dark cycle and $20^{\circ} \mathrm{C}$ day and $16^{\circ} \mathrm{C}$ night temperatures, supplied with cool, white fluorescent lamps. The plants were fertilized every two weeks.

\section{Fungal inoculum preparation, inoculation, and sampling procedures.}

Fusarium graminearum isolate $\mathrm{Fg} 4$ was used to introduce FHB into wheat. The procedures for pathogen preparation and $F$. graminearum inoculation were previously described by $\mathrm{Li}$ and Yen (2008). Basically, F. graminearum spores were suspended in sterile water and were filtered through four sterile cheesecloth layers. The first flowering floret of randomly chosen plants was either challenged with water-suspension of Fusarium graminearum macroconidia (80,000 to 100,000 conidia per milliliter) or with water as the control. The inoculated spikes were immediately covered with plastic bags for 4 to $96 \mathrm{~h}$ to maintain the humidity, and the plants were grown under a 16-h light and 8-h dark cycle and $20^{\circ} \mathrm{C}$ day and $16^{\circ} \mathrm{C}$ night temperatures. For eQTL study in RIL, at least three spikelets were inoculated per treatment per line, and only the first flowering spikelet was selected for inoculation for each plant. The inoculated spikelets were covered with plastic bags for $24 \mathrm{~h}$. For FDK evaluation, the same criteria were used as for the eQTL study, but no mock-inoculation was conducted. For the gene expression dynamic study, samples were collected at 4, 8, 12, 24, and 48 hai from Sumai 3 and Y1193-06 and at 12, 24, 48, 72, and 96 hai from the two NIL. At least ten spikelets were inoculated per treatment per line per time point, and the inoculated spikelets were covered with plastic bags for 4 to 96 , respectively, and also, only the first flowering spikelet was selected for each plant.

All plant samples were immediately put into liquid nitrogen after collection and were kept at $-80^{\circ} \mathrm{C}$ until use. Data for estimating the FHB DI and diseased kernels were also collected as described by Li and Yen (2008). All experiments were conducted in 2010 and then repeated in 2011.

\section{RNA isolation and real-time $q P C R$.}

RNA extraction was conducted using TRIzol RNA isolation reagents (Invitrogen, Carlsbad, CA, U.S.A.). The quality and quantity of the RNA samples were measured using a NanoDrop ND-1000 UV-Vis spectrophotometer (Wilmington, DE, U.S.A.). To assemble the resistant and the susceptible bulks, an equal amount of RNA from each line of the resistant or the susceptible subpopulation (three duplicated samples per line) were mixed together. Reverse transcription was conducted with RNA samples from all 80 RIL, the two bulks, and the two NIL lines at time points of interest. Briefly, $2 \mu \mathrm{g}$ of RNA per sample were used for cDNA synthesis in a $20-\mu$ l reaction using Superscript III enzyme (Invitrogen) with oligo(dT) ${ }_{15}$ primer (Promega, Madison, WI, U.S.A.), and then, a $5 \times$ dilution was made. For real-time qPCR, $1 \mu \mathrm{l}$ of $5 \times$-diluted cDNA and $10-\mu 1$ reactions using SYBR Green I master mix were carried out on an ABI 7900 machine (ABI, Foster City, CA, U.S.A.) with $10 \mathrm{~min}$ at $94^{\circ} \mathrm{C}, 40$ cycles of $20 \mathrm{~s}$ at $94^{\circ} \mathrm{C}, 30 \mathrm{~s}$ at melting temperature, and $30 \mathrm{~s}$ at $72^{\circ} \mathrm{C}$, and then, $3 \mathrm{~min}$ at $72^{\circ} \mathrm{C}$. Wheat $\beta$-actin gene was used as the reference gene to normalize the cycle threshold $(\mathrm{Ct})$ value. The four time point samples for each line tested were run on the same plate. For each sample, three technical repeats were done. Fold changes were calculated with the $\Delta \Delta \mathrm{Ct}$ method (Livak et al. 2001).

\section{The bulk and NIL screening.}

A total of 406 FHB-related wheat EST functionally identified in Sumai 3 were investigated for their association with FHB-resistance QTL. A twofold change in expression was set as the threshold of differential expression. For each gene, comparison was first made to identify differential expression between the $F$. graminearum-inoculated and the related mockinoculated control for each bulk and NIL. The select genes were then compared between the two bulks and NIL. Genes that passed the bulk analyses in both 2010 and 2011 were selected for eQTL analysis. A gene that showed differential expression between the two bulks was further compared between the two NIL for its association with Fhbl.

\section{The eQTL analysis and physical mapping.}

Samples collected at 24 hai from the 80 RIL were used for eQTL mapping. The $\Delta \Delta \mathrm{Ct}$ value of a gene in each line was treated as phenotypic data. The phenotypic data for each gene were first tested for their normality using the package "nortest" (Gross 2012), which was based on the open-source statistical language R ( R Development Core Team 2006). For those largely biased data, box-cox transformations were conducted (Sakia 1992). The linkage map was generated using MapChart 2.1 (Voorrips 2002), based on genotype data formerly generated and described in Basnet and associates (2012). QTL mapping for FHB resistance was conducted with WinQTLCart version 2.5 (Wang et al. 2005). Composite interval mapping was performed with an arbitrarily set LOD $=2.5$. All QTL or eQTL claimed were validated in QTLNetwork-2.0 (Yang et al. 2007). Finally, eQTL candidates that were located less than $2 \mathrm{cM}$ apart were counted as one locus, and only those candidates that were detected in both years were recognized as an eQTL.

The physical locations of candidate genes were confirmed in Chinese Spring nulli-tetra lines. DNA samples isolated from young leaves of the nulli-tetra lines were provided by W. Li of South Dakota State University. Their quality and quantity were measured using a NanoDrop ND-1000 UV-Vis spectrophotometer. PCR reactions were carried out in an MJ Research PTC-200 thermal cycler in a total volume of $20 \mu \mathrm{l}$, with a final concentration of $200 \mu \mathrm{M}$ each dNTP, $1.5 \mathrm{mM}$ of $\mathrm{MgCl}_{2}, 0.5 \mu \mathrm{M}$ each primer, $1.5 \mathrm{U}$ of $\mathrm{Taq}$ polymerase, and 50 $\mathrm{ng}$ of the template DNA. PCR amplifications were run at $94^{\circ} \mathrm{C}$ for $5 \mathrm{~min}$, followed by 35 cycles of $94^{\circ} \mathrm{C}$ for $20 \mathrm{~s}, 60^{\circ} \mathrm{C}$ for $30 \mathrm{~s}, 72^{\circ} \mathrm{C}$ for $30 \mathrm{~s}$, and a final extension of $72^{\circ} \mathrm{C}$ for $3 \mathrm{~min}$. PCR products were separated on a $12 \%$ polyacrylamide gel, were stained with ethidium bromide, and were visualized by UV light. 


\section{ACKNOWLEDGMENTS}

We thank W. Li for providing DNA stocks of Chinese Spring nulli-tetra lines and B. Bernard and W. Berzonsky for comments on manuscripts and proofreading. This research was partially supported by funds from South Dakota Wheat Commission, South Dakota Agriculture Experiment Station and US Wheat and Barley Scab Initiative. Y. Yen developed the concept and designed the study, Y. Zhuang conducted real-time qPCR experiments and analyzed the data, A. Gala performed plant inoculations, and all three wrote the manuscript.

\section{LITERATURE CITED}

An, S. H., Sohn, K. H., Choi, H. W., Hwang, I. S., Lee, S. C., and Hwang, B. K. 2008. Pepper pectin methylesterase inhibitor protein CaPMEI1 is required for antifungal activity, basal disease resistance and abiotic stress tolerance. Planta 228:61-78.

Anderson, J. A., Stack, R. W., Liu, S., Waldron, B. L., Fjeld, A. D., Coyne, C., Moreno-Sevilla, P., Fetch, J. M., Song, Q. J., Cregan, P. B., and Frohberg, R. C. 2001. DNA markers for Fusarium head blight resistance QTLs in two wheat populations. Theor. Appl. Genet. 102:11641168.

Basnet, B., Glover, K., Ibrahim, A., Yen, Y., and Chao, S. 2012. A QTL on chromosome 2DS of 'Sumai 3' increases susceptibility to Fusarium head blight in wheat. Euphytica 186:91-101.

Bernardo, A., Bai, G., Guo, P., Xiao, K., Guenzi, A., and Ayoubi, P. 2007. Fusarium graminearum-induced changes in gene expression between Fusarium head blight-resistant and susceptible wheat cultivars. Funct. Integr. Genom. 7:69-77.

Bernardo, A., Ma, H., Zhang, D., and Bai, G. 2012. Single nucleotide polymorphism in wheat chromosome region harboring Fhbl for Fusarium head blight resistance. Mol. Breed. 29:477-488.

Bertini, L., Caporale, C., Testa, M., Proietti, S., and Caruso, C. 2009. Structural basis of the antifungal activity of wheat PR4 proteins. FEBS (Fed. Eur. Biochem. Soc.) Lett. 583:2865-2871.

Bertini, L., Leonardi, L., Caporale, C., Tucci, M., Cascone, N., Di Berardino, I., Buonocore, V., and Caruso, C. 2003. Pathogen-responsive wheat PR4 genes are induced by activators of systemic acquired resistance and wounding. Plant Sci. 164:1067-1078.

Brem, R. B., Yvert, G., Clinton, R., and Kruglyak, L. 2002. Genetic dissection of transcriptional regulation in budding yeast. Science 296:752755.

Bruce, R. J., and West, C. A. 1982. Elicitation of casbene synthetase activity in castor bean. The role of pectic fragments of the plant cell wall in elicitation by a fungal endopolygalacturonase. Plant Physiol. 69:1181-1188.

Brummell, D. A., and Harpster, M. H. 2001. Cell wall metabolism in fruit softening and quality and its manipulation in transgenic plants. Plant Mol. Biol. 47:311-339.

Buerstmayr, H., Lemmens, M., Hartl, L., Doldi, L., Steiner, B., Stierschneider, M., and Ruckenbauer, P. 2002. Molecular mapping of QTLs for Fusarium head blight resistance in spring wheat. I. Resistance to fungal spread (Type II resistance). Theor. Appl. Genet. 104:84-91.

Buerstmayr, H., Ban, T., and Anderson, J. A. 2009. QTL mapping and marker-assisted selection for Fusarium head blight resistance in wheat: A review. Plant Breed. 128:1-26.

Bushnell, W. R., Hazen, B. E., and Pritsch, C. 2003. Histology and physiology of Fusarium head blight. Pages 44-83 in: Fusarium Head Blight of Wheat and Barley. K. J. Leonard and W. R. Bushnell, eds. APS Press, St. Paul, MN, USA,

Cho, S.-H., Lee, J., Jung, K.-H., Lee, Y.-W., Park, J.-C., and Paek, N.-C. 2012. Genome-wide analysis of genes induced by Fusarium graminearum infection in resistant and susceptible wheat cultivars. J. Plant Biol. 55:64-72.

Creelman, R. A., and Mullet, J. E. 1997. Biosynthesis and action of jasmonates in plants. Ann. Rev. Plant Physiol. Plant Mol. Biol. 48:355381.

Cuomo, C. A., Guldener, U., Xu, J. R., Trail, F., Turgeon, B. G., Di Pietro, A., Walton, J. D., Ma, L. J., Baker, S. E., Rep, M., Adam, G., Antoniw, J., Baldwin, T., Calvo, S., Chang, Y. L., Decaprio, D., Gale, L. R., Gnerre, S., Goswami, R. S., Hammond-Kosack, K., Harris, L. J., Hilburn, K., Kennell, J. C., Kroken, S., Magnuson, J. K., Mannhaupt, G., Mauceli, E., Mewes, H. W., Mitterbauer, R., Muehlbauer, G., Münsterkötter, M., Nelson, D., O'Donnell, K., Ouellet, T., Qi, W., Quesneville, H., Roncero, M. I., Seong, K. Y., Tetko, I. V., Urban, M., Waalwijk, C., Ward, T. J., Yao, J., Birren, B. W., and Kistler, H. C. 2007. The Fusarium graminearum genome reveals a link between localized polymorphism and pathogen specialization. Science 317:1400-1402.

Cuthbert, P., Somers, D., and Brulé-Babel, A. 2007. Mapping of Fhb2 on chromosome 6BS: A gene controlling Fusarium head blight field resistance in bread wheat (Triticum aestivum L.). TAG Theor. Appl. Genet. 114:429-437.

Desjardins, A. E., and Hohn, T. M. 1997. Mycotoxins in plant pathogenesis. Mol. Plant-Microbe Interact. 10:147-152.

Doares, S. H., Syrovets, T., Weiler, E. W., and Ryan, C. A. 1995. Oligogalacturonides and chitosan activate plant defensive genes through the octadecanoid pathway. Proc. Natl. Acad. Sci. U.S.A. 92:4095-4098.

Feussner, I., and Wasternack, C. 2002. The lipoxygenase pathway. Ann. Rev. Plant Biol. 53:275-297.

Foroud, N. A., Ouellet, T., Laroche, A., Oosterveen, B., Jordan, M. C., Ellis, B. E., and Eudes, F. 2012. Differential transcriptome analyses of three wheat genotypes reveal different host response pathways associated with Fusarium head blight and trichothecene resistance. Plant Pathol. 61:296-314.

Gao, L., Fei, C., Zhou, L., and Zhong, W. 2005. Genetic analysis of resistance to wheat scab (Fusarium graminearum Schw) in Wangshubai. J. Triticeae Crops 25:5-9.

Gilbert, J., and Fernando, W. G. D. 2004. Epidemiology and biological control of Gibberella zeae/Fusarium graminearum. Can. J. Plant Pathol. 26:464-472.

Gottwald, S., Samans, B., Luck, S., and Friedt, W. 2012. Jasmonate and ethylene dependent defence gene expression and suppression of fungal virulence factors: Two essential mechanisms of Fusarium head blight resistance in wheat? BMC Genomics 13:369.

Gross J. 2012. nortest: Tests for Normality. R package version 1.0-1. cran.r-project.org/web/packages/nortest/index.html. Published online.

He, Z. H., Rajaram, S., Xin, Z. Y., and Huang, G. Z. 2001. A History of Wheat Breeding in China. Centro Internacional de Mejoramiento de Maíz y Trigo. Batan, Mexico.

Hejgaard, J., Jacobsen, S., Bjorn, S., and Kragh, K. M. 1992. Antifungal activity of chitin-binding PR-4 type proteins from barley grain and stressed leaf. FEBS (Fed. Eur. Biochem. Soc.) Lett. 307:389-392.

Hill-Ambroz, K., Webb, C. A., Matthews, A. R., Li, W., Gill, B. S., and Fellers, J. P. 2006. Expression analysis and physical mapping of a cDNA library of Fusarium head blight infected wheat spikes. Crop Sci. 46:S15-S26.

Jia, H., Cho, S., and Muehlbauer, G. J. 2009. Transcriptome analysis of a wheat near-isogenic line pair carrying Fusarium head blight-resistant and -susceptible alleles. Mol. Plant-Microbe Interact. 22:1366-1378.

Kang, Z., and Buchenauer, H. 2000. Cytology and ultrastructure of the infection of wheat spikes by Fusarium graminearum. Mycol. Res. 104:1083-1093.

Kruger, W. M., Pritsch, C., Chao, S., and Muehlbauer, G. J. 2002. Functional and comparative bioinformatic analysis of expressed genes from wheat spikes infected with Fusarium graminearum. Mol. PlantMicrobe Interact. 15:445-455.

Lemmens, M., Scholz, U., Berthiller, F., Asta, C. D., Koutnik, A., Schuhmacher, R., Adam, G., Buerstmayr, H., Mesterhazy, A., Krska, R., and Ruckenbauer, P. 2005. The ability to detoxify the mycotoxin deoxynivalenol colocalizes with a major quantitative trait locus for $\mathrm{Fu}$ sarium head blight resistance in wheat. Mol. Plant-Microbe Interact. 18:1318-1324.

Li, G., and Yen, Y. 2008. Jasmonate and ethylene signaling pathway may mediate Fusarium head blight resistance in wheat. Crop Sci. 48:18881896.

Liu, S., Pumphrey, M. O., Gill, B. S., Trick, H. N., Zhang, J. X., Dolezel, J., Chalhoub, B., and Anderson, J. A. 2008. Toward positional cloning of Fhb1, a major QTL for Fusarium head blight resistance in wheat. Cereal Res. Comm, 36:195-201.

Lionetti, V., Raiola, A. Camardella, L., Giovane, A., Obel, N., Pauly, M., Favaron, F., Cervone, F., and Bellincampi, D. 2004. Overexpression of pectin methylesterase inhibitors in Arabidopsis restricts fungal infection by Botrytis cinerea. Plant Physiol 143:1871-1880.

Livak, K. J., and Schmittgen, T. D. 2001. Analysis of relative gene expression data using real-time quantitative PCR and the $2^{-\Delta \Delta \mathrm{Ct}}$ method. Methods 25:402-408.

Marty, P., Jouan, B., Bertheau, Y., Vian, B., and Goldberg, R. 1997. Charge density in stem cell walls of Solanum tuberosum genotypes and susceptibility to blackleg. Phytochemistry 44:1435-1441.

McMullen, M., Jones, R., and Gallenberg, D. 1997. Scab of wheat and barley: A re-emerging disease of devastating impact. Plant Dis. 81:1340-1348.

Mesterhazy, A. 1995. Types and components of resistance to Fusarium head blight of wheat. Plant Breed. 114:377-386.

Paranidharan, V., Abu-Nada, Y., Hamzehzarghani, H., Kushalappa, A. C., Mamer, O., Dion, Y., Rioux, S., Comeau, A., and Choiniere, L. 2008. Resistance-related metabolites in wheat against Fusarium graminearum and the virulence factor deoxynivalenol (DON). Botany 86:1168-1179.

Parry, D. W., Jenkinson, P., and McLeod, L. 1995. Fusarium ear blight 
(scab) in small grain cereals-A review. Plant Pathol. 44:207-238.

Pritsch, C., Muehlbauer, G. J., Bushnell, W. R., Somers, D. A., and Vance, C. P. 2000. Fungal development and induction of defense response genes during early infection of wheat spikes by Fusarium graminearum. Mol. Plant-Microbe Interact. 13:159-169.

Pumphrey, M. O., Bernardo, R., and Anderson, J. A. 2007. Validating the QTL for Fusarium head blight resistance in near-isogenic wheat lines developed from breeding populations. Crop Sci. 47:200-206.

R Development Core Team. 2006. R: A language and environment for statistical computing. R Foundation for Statistical Computing, Vienna.

Rocchi, V., Janni, M., Bellincampi, D., Giardina, T., and D'Ovidio, R. 2011. Intron retention regulates the expression of pectin methyl esterase inhibitor (Pmei) genes during wheat growth and development. Plant Biol. 14:365-373

Sakia, R. M. 1992. The Box-Cox transformation technique: A review. The Statistician 41:169-178.

Schaller, F., Schaller, A., and Stintzi, A. 2004. Biosynthesis and metabolism of jasmonates. J. Plant Growth Regul. 23:179-199.

Schroeder, H. W., and Christensen, J. J. 1963. Factors affecting resistance of wheat to scab caused by Gibberella zeae. Phytopathology 53:831-838.

Seong, K.-Y., Zhao, X., Xu, J., Güldener, U., and Kistler, H. C. 2008. Conidial germination in the filamentous fungus Fusarium graminearum. Fungal Genet. Biol. 45: 389-399.

Snijders, C. H. A. 1990. Fusarium head blight and mycotoxin contamination of wheat, a review. Eur. J. Plant Pathol. 96:187-198.

Somers, D. J., Fedak, G., and Savard, M. 2003. Molecular mapping of novel genes controlling Fusarium head blight resistance and deoxynivalenol accumulation in spring wheat. Genome 46:555-564.

Trail, F., Xu, J. R., San Miguel, P., Halgren, R. G., and Kistler, H. C. 2003. Analysis of expressed sequence tags from Gibberella zeae (anamorph
Fusarium graminearum). Fungal Genet. Biol. 38:187-197.

Van Ginkel, M., Van der Schaar, W., Yang, Z., and Rajaram, S. 1996. Inheritance of resistance to scab in two wheat cultivars from Brazil and China. Plant Dis. 80:863-867.

Volpi, C., Janni, M., Lionetti, V., Bellincampi, D., Favaron, F., and D'Ovidio, R. 2011. The ectopic expression of a pectin methyl esterase inhibitor increases pectin methyl esterification and limits fungal diseases in wheat. Mol. Plant-Microbe Interact. 24:1012-1019.

Voorrips, R. E. 2002. MapChart: Software for the graphical presentation of linkage maps and QTLs. J. Hered. 93:77-78.

Wang, S., Basten, C. J., and Zeng, Z. B. 2005 Windows QTL cartographer 2.5. Department of Statistics North Carolina State University, Raleigh, NC, U.S.A.

Wilde, F., Korzun, V., Ebmeyer, E., Geiger, H. H., and Miedaner, T. 2007. Comparison of phenotypic and marker-based selection for Fusarium head blight resistance and DON content in spring wheat. Mol. Breed. 19:357-370.

Yamashita, S., Wakazono, K., Nomoto, T., Tsujino, Y., Kuramoto, T., and Ushijima, T. 2005. Expression quantitative trait loci analysis of 13 Genes in the rat prostate. Genetics 171:1231-1238.

Yang, J., Zhu, J., and Williams, R. W. 2007. Mapping the genetic architecture of complex traits in experimental populations. Bioinformatics 23:1527-1536.

Zhou, M., Hayden, M., Zhang, Z., Lu, W., and Ma, H. 2010. Saturation and mapping of a major Fusarium head blight resistance QTL on chromosome 3BS of Sumai 3 wheat. J. Appl. Genet. 51:19-25.

Zhou, W. C., Kolb, F. L., Bai, G. H., Domier, L. L., and Yao, J. B. 2002. Effect of individual Sumai 3 chromosomes on resistance to scab spread within spikes and deoxynivalenol accumulation within kernels in wheat. Hereditas 137:81-89. 